Variance estimation methods for health expectancy by relative socio-economic status

Peer-reviewed author version

Abatih, Emmanuel; Van Oyen, Herman; Bossuyt, Nathalie \& BRUCKERS, Liesbeth (2008) Variance estimation methods for health expectancy by relative socio-economic status. In: EUROPEAN JOURNAL OF EPIDEMIOLOGY, 23(4). p. 243-249.

DOI: $10.1007 / \mathrm{s} 10654-008-9227-5$

Handle: http://hdl.handle.net/1942/8149 


\title{
Variance estimation methods for health expectancy by relative socio-economic status
}

\author{
Emmanuel Abatih $\cdot$ Herman Van Oyen \\ Nathalie Bossuyt $\cdot$ Liesbeth Bruckers
}

Received: 26 January 2007 / Accepted: 23 January 2008

(C) Springer Science+Business Media B.V. 2008

\begin{abstract}
In many studies, health expectancies (HE) by relative socio-economic status have been calculated but the estimation of confidence intervals and the performance of tests of significance for differences in HE between subpopulations have been impeded by lack of variance estimation methods. Also in most scenarios, the sampling designs of the surveys from which prevalence of ill-health conditions are obtained have been ignored. This paper aims at presenting variance estimation techniques such as the bootstrap and the delta method taking account of the survey design. The study suggests that using the raw survey data and the Delta method while accounting for the survey design, gives better estimates for the variance compared to the bootstrap method and therefore is a highly recommended method for variance estimation of HE by relative socio-economic status.
\end{abstract}

Keywords Survey design - Bootstrap · Delta method . Variance $\cdot$ Prevalence $\cdot$ Sullivan

\section{Introduction}

Health expectancy (HE) is a composite health indicator combining information on morbidity and mortality. It describes how long a person can expect to live in good health if the current age-specific mortality and morbidity

E. Abatih $(\bowtie) \cdot$ L. Bruckers

Center for Statistics, University of Hasselt, Universitaire

Campus - building D, Diepenbeek 3590, Belgium

e-mail: ena@life.ku.dk

E. Abatih $\cdot$ H. Van Oyen - N. Bossuyt

Scientific Institute of Public Health, Epidemiology Unit, Juliette,

Wytsmanstraat 14, Brussels 1050, Belgium conditions were to prevail in future [1]. The term "health expectancy" is used to refer to health expectancies in general, without specifying the indicator of morbidity it is based on (long standing illness, perceived poor general health status and functional limitations). The notion of health expectancy was first put forward by Sanders as early as 1964 [2] and Sullivan proposed a method of calculation in 1971 [3]. Since then, HEs have been increasingly used in different areas of health policy; such as monitoring and evaluating the health status of the population, studying health inequalities among different population groups or allocating resources in the short term [4]. Socio-economic gradients in HE have been consistently described in several countries [5-10]. The relative index of inequality has been proposed as a measure of socio-economic inequality in mortality rates or disease prevalence rates on a relative scale and Fieller's method for estimating confidence intervals about the estimated relative index of inequality was suggested [11].

However, the few studies that have examined socioeconomic inequalities in $\mathrm{HE}$ on a relative scale have been based on aggregated data (prevalence by age, sex, educational level and region) without variance estimation and statistical testing of the inequalities [7, 9]. Further, the sampling designs of the health interview surveys from which the prevalence of ill-health conditions are obtained have not been taken into account.

This paper describes methods for estimating the variances of the HEs and the size of the socio-economic differences in the HEs calculated using aggregated data as well as the raw survey data from which prevalence of ill-health conditions are obtained. After a presentation of the bootstrap method and the delta method, a case study is presented in which the morbidity is defined in terms of functional limitations: the Disability Free Life Expectancy (DFLE) [12]. 


\section{Materials and methods}

Data sources

The calculation of HEs and their variances requires data on both mortality and morbidity. Mortality data can be obtained from national databases and also from the World Health Organisation (WHO) database which contains number of deaths by year and cause for each member state. Mortality statistics are considered reliable and cover the total population. Morbidity data on the other hand are usually obtained from national health interview surveys.

\section{Measure of morbidity}

Measures of morbidity vary from global perception of one's own general health to more complex indices measuring the consequences of disease or illness on one's ability to carry out everyday activities. Examples of such measures include amongst others perceived general health status, limiting or long-standing illnesses, and functional limitations.

\section{Measure of socio-economic status}

The indicators of socio-economic status (SES) are educational level, income, occupational class or a composite of these dimensions. Educational level has been the indicator of choice for most mortality and health studies in both Demography and Epidemiology, as it appears to have several advantages over the others. Firstly, educational attainment can be measured in a comparable way for almost all individuals. Secondly, it has a more accurate response and a lower non-response rate. Thirdly, it is relatively stable over time and finally, health impairments that emerge in adulthood rarely affect educational attainment since it is normally completed by the early adult years, around age 25 [13].

An important methodological issue is the cohort comparability of the measures of socio-economic status; the meaning of specific levels of socio-economic status differ between birth cohorts both nationally and internationally [7].

Measurement of inequalities in health expectancy

The calculation of health expectancies discussed in this paper follows Sullivan's method [3]. It is based on the life table [14], which describes the survival experience of a real or hypothetical group of people followed from birth or from other ages in their lifetime. The HE is given by:
$H \hat{E}_{i}=\frac{\left[\sum_{i=x}^{w}\left(1-\pi_{i}\right) * L_{i}\right]}{l_{x}}$

where $\pi_{i}$ is the prevalence of the ill-health condition for age group $i$ (obtained from health interview surveys), $L_{i}$ is the number of person years lived in the age group, $l_{x}$ the number of persons surviving at the beginning of the age interval (obtained from the abridged current life table), and $w$ the final age category in the life table.

The variance of the HE is calculated as $[3,15]$ :

$\operatorname{var}\left(H E_{i}\right)=\frac{1}{l_{x}^{2}} \sum_{i=x}^{w} L_{i}^{2} * \operatorname{var}\left(\pi_{i}\right)$

where the variance of the prevalence is given by:

$\operatorname{var}\left(\pi_{i}\right)=\frac{\pi_{i} *\left(1-\pi_{i}\right)}{N}$

In equation 2.2, it is assumed that the prevalence follows a Bernouilli distribution.

One approach to determine the inequality in health expectancy is to compare the health expectancies of people at the highest and at the lowest level of the socio-economic scale [7]. However, only the extreme groups are compared and the sizes of these two groups are not the same. The association throughout all levels of education, including the intermediate groups and the sizes of the groups are not taken into account. Also the sense of specific SES positions vary between birth cohorts. These shortcomings are avoided by using a regression-based method first developed by Pamuk in 1985 [16] and later used by Sihvonen et al. [7], Hayes and Berry [11] and Bossuyt et al. [9]. In this method, an individual's position in the socio-economic hierarchy is transformed into his/her relative position on a socio-economic scale (continuous scale from 0 to 1 ) in a manner similar to the relative index of inequality in mortality rates and disease prevalence rates [11]. For example if the lowest income group consists of $20 \%$ of the population, the relative position of its members is $0 \leq \mathrm{x}<0.2$, the average being 0.1 . If the next group in the hierarchy comprises of $35 \%$ of people, its member's relative position is $0.2 \leq \mathrm{x}<0.55$ on average 0.375 , and so on. Thus the socio-economic classification becomes independent and more robust in the face of differences in its definition. The inequality in HE is now defined as the difference between the virtual top (1) and bottom (0) of this scale. This gives the maximum difference in $\mathrm{HE}$ that could be observed for a given population.

The regression methods assumed a linear relationship between the morbidity and the relative position on the social hierarchy. This assumption is only for the sake of simplicity because most often, the relationships may appear to be nonlinear. However, models that best fit such data 
(such as polynomials or other non-linear models) are not to be trusted due to the small sample sizes (resulting from the number of socio-economic categories) involved and the fact that the model could be under-identified; i.e. there are more parameters to be estimated than there are observations. The major drawback of this approach is its inability to yield valid and reliable estimates for the variances of the prevalences and therefore the variance of the HE. We present two methods to estimate the variances of the health expectancies at both ends (virtual top (relative position $($ relpos $)=1)$ and bottom (relpos $=0)$ ) of the relative social hierarchy and the variance of the inequality in HE, defined as the difference between the HE at the top and HE at the bottom of the social hierarchy.

Estimation of the variance of HE using the bootstrap method

Assuming a linear relationship between the prevalence of the ill-health condition and the relative position on the socio-economic scale [7], the following model is fitted for each age group (5 or 10 year age intervals):

$\pi=\alpha+\beta * x+\varepsilon$

where $\alpha$ and $\beta$ are parameters to be estimated from the data, $\pi$ is the prevalence and $x$ is the relative position on the social hierarchy.

The prevalences are estimated based on different number of persons in each educational category thus making them more precisely estimated for groups with more persons. Thus weighted least squares are used to maximize the efficiency of parameter estimation. The weights are the relative sizes of the SES levels in each age group based on the population numbers as estimated from censuses if such data are available or from surveys otherwise. The bootstrapping was done using the following steps [17]:

1. Within each age and sex stratum, use the observed prevalence of the ill-health condition for each socioeconomic position as the mean of a Bernoulli distribution i.e. $p^{1}, p^{2}, p^{3}, \ldots, p^{s}$ where $\mathrm{S}$ is the number of socio-economic positions in the study.

2. Sample $m^{1}, m^{2}, m^{3}, \ldots, m^{s}$ observations from the Bernoulli distribution where the $m$ 's are the sizes of the socio-economic groups considered. Calculate the means of the newly generated samples and call them $p^{* 1}, p^{* 2}, p^{* 3}, \ldots, p^{* s}$. Note that $p^{* 1}$ is estimated based on $m^{1}, p^{* 2}$ on $m^{2}$ and so on.

3. Fit model (3) using the newly generated prevalences of the ill-health condition from step 2 above as the response variable
4. Use fitted model from step 3 to predict the prevalence of those at the virtual top (Relpos $=1$ ) and the virtual bottom (Relpos $=0$ ) of the socio-economic scale.

5. Repeat steps $2-4 n$ times so that we end up with $n$ predicted prevalences for the lowest and highest positions of the socio-economic scale for each age group.

6. The generated prevalences are then used to calculate $n$ HEs using (1).

7. The distribution of these $n$ HEs is obtained and used to calculate the HEs (mean of the distribution of estimates) and their variances (variance of the distribution of the estimates).

It is suggested that 200 bootstrap samples are good enough to yield correct variance estimates [17]. Studentized bootstrap confidence intervals, which give better coverage, are then calculated for the HEs [17].

Estimation of the variance of HE using the delta method

In this method, the survey logistic regression model which takes full account of the sampling design of the survey (sampling weights, clustering and stratification factors), is used to obtain the prevalence of the ill-health condition and the variance of the estimated log odds of the prevalence. Since a direct computation of the variance of the prevalence is not feasible, the delta method [18] was applied. Given that the log odds and the prevalence have a direct relationship, the delta method approximates the variance of the prevalence based on that of the log odds through a simple transformation. The computations are given in Appendix A.

Using the predicted prevalence and (1), the HE of persons at the top and bottom of the socio-economic scale is obtained. Once the variance of the prevalence is obtained, the variance of the $\mathrm{HE}$ is obtained using the estimated variance of the prevalence and (2.1).

Confidence intervals (95\%) for the estimated HEs are obtained using the normal approximation to the binomial i.e. C.I. $=H \hat{E} \pm s e(H \hat{E}) * 1.96$ with $s e(H \hat{E})$ being the standard error of the health expectancy.

\section{Confidence intervals for inequalities in $\mathrm{HE}$}

After computing the HE using either of the two methods (Bootstrap or Delta method) and computing differences in HEs, the question remains whether the observed differences are due to chance processes alone. Confidence intervals were used as an alternative to the Z-score test [19, 20] since they 
contain the information of a significant test alongside information on accuracy of estimation and interpretation. To construct $95 \%$ confidence intervals for the inequalities in $\mathrm{HE}$, assume that $H \hat{E}_{1}$ stands for $\mathrm{HE}$ at the top of the socioeconomic scale and $H \hat{E}_{2}$ for $\mathrm{HE}$ at the bottom of the socioeconomic scale for males at a specific age for example. The variance of the difference in HEs can be estimated based on the Cauchy Swartz inequality [21] as:

$\operatorname{var}\left(H \hat{E}_{1}-H \hat{E}_{2}\right) \leq\left[\sqrt{\operatorname{var}\left(H \hat{E}_{1}\right)}+\sqrt{\operatorname{var}\left(H \hat{E}_{2}\right)}\right]^{2}$

where $\sqrt{\operatorname{var}\left(H \hat{E}_{1}\right)}$ and $\sqrt{\operatorname{var}\left(H \hat{E}_{2}\right)}$ represent the standard errors of $H \hat{E}_{1}$ and $H \hat{E}_{2}$.

The $95 \%$ confidence intervals (lower and upper) may be conservatively approximated by:

$\left(H \hat{E}_{1}-H \hat{E}_{2}\right) \pm 1.96 * \sqrt{\operatorname{var}\left(H \hat{E}_{1}-H \hat{E}_{2}\right)}$

In a similar manner, the significance of the differences in socio-economic gradient between subpopulations is tested.

The hypothesis of equality of HEs is rejected if the $95 \%$ confidence interval fails to include zero.

\section{Case study}

\section{Summary}

The aim is to evaluate the performance of the Bootstrap and the Delta method in the estimation of the variance of the Disability Free Life Expectancy (DFLE) and testing of significance for differences in DFLE between subgroups in the Belgian population. The DFLE is a special case of the health expectancy where the functional disability indictor is applied.

Mortality data by level of education was derived from the Belgian National Mortality Database (NMD), a 5year follow up of the 1991 census. Data on the prevalence of disability was obtained from the Belgian National Health Interview Survey (HIS) conducted in 1997. The survey was based on a representative sample of the Belgian population and used a multistage sampling design procedure [22].

Only persons aged between 25 and 74 years were included in this exercise, as the younger people had not yet completed their education and people older than 75 were more likely to be institutionalized and interviewers had problems reaching them. Since the estimated DFLE is compared not only between the Flemish and the Walloon regions but also between the socio-economic groups within these regions, the number of persons in certain age groups was very small. This was accounted for by constructing abridged life tables with 10 years age intervals.
The prevalence of disability was measured using the WHO instrument including activities of daily living functions (e.g. transfer in and out bed or in and out a chair, dressing, feeding etc.) [23]. Due to the additional advantage of being the only instrument in both the NMD and the HIS, educational level, was taken as the measure of choice for the socio-economic status. A four-category classification of education based on individual's own educational level was used on the basis of the highest educational attainment:

(1) Primary education or less

(2) Lower secondary education (lower vocational/technical and general education)

(3) Higher secondary education (higher vocational/technical and general education)

(4) Higher education (3 years +: college and university)

The relative position of each level on the SES-scale was determined for each gender specific 10-years birth cohort as previously described. The association between prevalence of disability and the relative position on the SES scale by 10 -year age group was estimated using the two regression-based methods described above. Partial DFLE for ages 25-74 were calculated by using the prevalence of disability, predicted by the regression models for those at the top and the bottom of the socio-economic scale in each 10 -year age group and Sullivan's method. The variances of the DFLE were calculated using the bootstrap method and the Delta method. For the bootstrap method the data was aggregated by age-group and gender.

\section{Results}

The bootstrap and delta methods were used to obtain estimates of the DFLEs and their variances by relative socio-economic status for males and females in the Flemish and Walloon regions of Belgium.

In general, the estimated DFLEs from both methods were comparable with a few exceptions. For example (Table 1) at age 25 years, the DFLE and its variance were 34.43 years and 3.332 respectively for Flemish females at the lowest SES position as given by the bootstrap method (columns 2 and 3), whereas for the delta method the DFLE and its variance were 28.30 years and 0.907 respectively (columns 6 and 7). Similar differences in DFLE between the methods were observed for the other age groups. On the other hand, at the highest position of the social hierarchy, the estimates for the DFLEs were similar for both methods at all ages. The variances were however larger for the bootstrap method compared to that of the delta method.

For Flemish males and for Walloon males and females, the DFLEs appeared also to be similar with variances from 
Table 1 DFLE $_{25-74}$ by relative socio-economic position; Comparison of results from the Bootstrap and delta methods for Flemish and Walloon males and females for various age groups, Belgium 1997

\begin{tabular}{|c|c|c|c|c|c|c|c|c|}
\hline \multirow[t]{3}{*}{ At age } & \multicolumn{4}{|l|}{ Bootstrap } & \multicolumn{4}{|c|}{ Delta method } \\
\hline & \multicolumn{2}{|l|}{ Bottom } & \multicolumn{2}{|l|}{ Top } & \multicolumn{2}{|l|}{ Bottom } & \multicolumn{2}{|l|}{ Top } \\
\hline & DFLE $_{25-74}$ & Variance & DFLE $_{25-74}$ & Variance & DFLE $_{25-74}$ & Variance & DFLE $_{25-74}$ & Variance \\
\hline \multicolumn{9}{|c|}{ Flemish females } \\
\hline 25 & 34.43 & 3.332 & 39.97 & 1.120 & 28.30 & 0.907 & 41.93 & 0.221 \\
\hline 35 & 24.75 & 3.232 & 30.92 & 1.072 & 20.13 & 0.825 & 32.42 & 0.217 \\
\hline 45 & 17.77 & 2.571 & 21.40 & 0.939 & 12.95 & 0.661 & 23.22 & 0.210 \\
\hline 55 & 10.98 & 1.943 & 12.68 & 0.731 & 6.86 & 0.455 & 14.49 & 0.194 \\
\hline 65 & 3.53 & 1.024 & 6.12 & 0.438 & 2.72 & 0.181 & 6.72 & 0.126 \\
\hline \multicolumn{9}{|c|}{ Flemish males } \\
\hline 25 & 29.44 & 2.761 & 43.59 & 0.638 & 30.16 & 0.761 & 42.09 & 0.130 \\
\hline 35 & 20.79 & 2.432 & 34.11 & 0.523 & 21.75 & 0.713 & 32.62 & 0.129 \\
\hline 45 & 12.22 & 2.278 & 24.67 & 0.486 & 14.22 & 0.612 & 23.45 & 0.127 \\
\hline 55 & 6.09 & 1.722 & 15.78 & 0.398 & 7.76 & 0.471 & 14.81 & 0.123 \\
\hline 65 & 2.24 & 0.956 & 7.39 & 0.289 & 3.18 & 0.224 & 7.04 & 0.090 \\
\hline \multicolumn{9}{|c|}{ Walloon females } \\
\hline 25 & 24.86 & 2.261 & 41.47 & 1.034 & 25.30 & 0.855 & 39.84 & 0.240 \\
\hline 35 & 16.83 & 2.075 & 32.36 & 0.956 & 17.58 & 0.750 & 30.50 & 0.235 \\
\hline 45 & 10.96 & 1.473 & 23.28 & 0.876 & 11.00 & 0.556 & 21.55 & 0.224 \\
\hline 55 & 3.83 & 1.199 & 15.11 & 0.733 & 5.61 & 0.359 & 13.21 & 0.204 \\
\hline 65 & 1.03 & 0.450 & 7.09 & 0.428 & 2.17 & 0.128 & 6.04 & 0.121 \\
\hline \multicolumn{9}{|c|}{ Walloon males } \\
\hline 25 & 27.68 & 2.230 & 40.45 & 0.725 & 27.00 & 0.729 & 39.80 & 0.161 \\
\hline 35 & 19.83 & 2.084 & 30.79 & 0.726 & 19.02 & 0.664 & 30.52 & 0.160 \\
\hline 45 & 12.90 & 1.514 & 21.70 & 0.629 & 12.07 & 0.535 & 21.65 & 0.159 \\
\hline 55 & 6.88 & 1.396 & 13.24 & 0.616 & 6.34 & 0.391 & 13.47 & 0.155 \\
\hline 65 & 4.21 & 0.734 & 5.68 & 0.459 & 2.55 & 0.167 & 6.34 & 0.112 \\
\hline
\end{tabular}

the bootstrap being larger than those from the delta method.

The performance of both methods in testing significance of the inequalities in DFLE between the top and bottom of the social hierarchy was also assessed. The two methods agreed quite well on testing the significance of observed differences in DFLE between the lowest and the highest position on the socio-economic scale for Flemish males and Walloon females whereas some disagreements were displayed for the case of Flemish females and Walloon males (Table 2). For example at age 25 years, the inequality in DFLE for Flemish males was statistically significant at the $5 \%$ level of significance (C.I. : $(9.33,18.97)$ does not include zero) (column 4) according to the bootstrap method. The same conclusion was obtained using the delta method (column 7). The same trends were observed at all other ages. On the other hand, for Flemish females, whereas the bootstrap method yielded non-significant inequalities in DFLE at all ages except 35 years, the delta method indicated significant differences in the DFLE at all ages.
Just as for Flemish males, Walloon males and females presented significant differences in DFLE between the top and the bottom of the socio-economic scale for both methods at all ages (C.Is. Do not include zero) except 65 years where the bootstrap yielded a non-significant inequalities in the DFLE for Walloon males (C.I.: $(-1.54$, 4.48)) does include zero).

\section{Discussion}

The results obtained from this case study highlight two key facts; firstly that the sampling design of the survey from which prevalence of ill-health conditions are obtained should be taken into account and also that where possible, the raw survey data should be used rather than their aggregated counterpart. The bootstrap, which is a very powerful method for estimating variances for small sample size data, yielded variance estimates that were larger than those of the logistic regression method (delta method). The 
Table 2 Inequalities in DFLE $25-74$ by relative socio-economic position; Comparison of results from the Bootstrap and delta methods for Flemish and Walloon males and females for various age groups, Belgium 1997

\begin{tabular}{|c|c|c|c|c|c|c|}
\hline \multirow[t]{2}{*}{ At age } & \multicolumn{3}{|l|}{ Bootstrap } & \multicolumn{3}{|l|}{ Delta method } \\
\hline & Inequality in DFLE $_{25-74}$ & Approx se & $95 \%$ confidence limits & Inequality in DFLE $_{25-74}$ & Approx s.e & $95 \%$ confidence limits \\
\hline \multicolumn{7}{|c|}{ Flemish females } \\
\hline 25 & 5.54 & 2.884 & $(-0.11,11.19)$ & 13.63 & 1.422 & $(10.84,16.42)$ \\
\hline 35 & 6.17 & 2.833 & $(0.62,11.72)$ & 12.29 & 1.374 & $(9.60,14.98)$ \\
\hline 45 & 3.64 & 2.572 & $(-1.40,8.68)$ & 10.27 & 1.272 & $(7.78,12.76)$ \\
\hline 55 & 1.70 & 2.249 & $(-2.71,6.11)$ & 7.63 & 1.115 & $(5.44,9.82)$ \\
\hline 65 & 2.58 & 1.674 & $(-0.70,5.86)$ & 4.00 & 0.781 & $(2.47,5.53)$ \\
\hline \multicolumn{7}{|c|}{ Flemish males } \\
\hline 25 & 14.15 & 2.460 & $(9.33,18.97)$ & 11.93 & 1.232 & $(9.52,14.34)$ \\
\hline 35 & 13.33 & 2.282 & $(8.86,17.80)$ & 10.87 & 1.203 & $(8.51,13.23)$ \\
\hline 45 & 12.45 & 2.206 & $(8.13,16.77)$ & 9.23 & 1.139 & $(7.00,11.46)$ \\
\hline 55 & 9.69 & 1.943 & $(5.88,13.50)$ & 7.06 & 1.037 & $(5.03,9.09)$ \\
\hline 65 & 5.15 & 1.515 & $(2.18,8.12)$ & 3.85 & 0.773 & $(2.33,5.37)$ \\
\hline \multicolumn{7}{|c|}{ Walloon females } \\
\hline 25 & 16.61 & 2.521 & $(11.67,21.55)$ & 14.54 & 1.415 & $(11.77,17.31)$ \\
\hline 35 & 15.53 & 2.418 & $(10.79,20.27)$ & 12.92 & 1.351 & $(10.27,15.57)$ \\
\hline 45 & 12.31 & 2.150 & $(8.10,16.52)$ & 10.56 & 1.219 & $(8.17,12.95)$ \\
\hline 55 & 11.28 & 1.952 & $(7.45,15.11)$ & 7.61 & 1.051 & $(5.55,9.67)$ \\
\hline 65 & 6.06 & 1.324 & $(3.46,8.66)$ & 3.87 & 0.705 & $(2.49,5.25)$ \\
\hline \multicolumn{7}{|c|}{ Walloon males } \\
\hline 25 & 12.78 & 2.345 & $(8.18,17.38)$ & 12.80 & 1.256 & $(10.34,15.26)$ \\
\hline 35 & 10.96 & 2.296 & $(6.46,15.46)$ & 11.51 & 1.215 & $(9.13,13.89)$ \\
\hline 45 & 8.80 & 2.023 & $(4.83,12.77)$ & 9.58 & 1.130 & $(7.37,11.79)$ \\
\hline 55 & 6.36 & 1.966 & $(2.51,10.21)$ & 7.13 & 1.020 & $(5.13,9.13)$ \\
\hline 65 & 1.47 & 1.534 & $(-1.54,4.48)$ & 3.79 & 0.744 & $(2.33,5.25)$ \\
\hline
\end{tabular}

bootstrap method did not take full account of the survey sampling design and used aggregated data (proportions) whereas the delta method took full account of the sampling design of the survey and used the raw survey data. Also, similar to previous publications $[7,9,11]$, a linear relationship between the disability prevalence and the relative position on the socio-economic scale was assumed in the bootstrap method but this was just for the sake of simplicity as most of the relationships appeared to be non-linear. Further attempts to select models that best described the relationship between the prevalence and the socio-economic position (such as polynomials) were futile with the bootstrap as it is completely automatic and was based only on 4 observations, the 4 levels of education considered. This was not the case with the logistic regression method which does not depend on the number of levels in the social hierarchy.

The results of the bootstrap method can be improved by increasing the sample size; this means increasing the number of categories for the educational level. With many points, the nature of the relationship between prevalence and socio-economic status could be defined with more certainty and the variance estimates could be more correct. The logistic regression model on which the delta method is based on the other hand did not make any linearity assumption between the prevalence and the relative socioeconomic positions. However, the estimated DFLE from the two methods were quite similar except for Flemish females. Due to the aforementioned drawbacks, the estimated variances from the bootstrap turned out to be larger than those from the survey logistic regression method and also resulted in different conclusions in comparison with the survey logistic regression procedure. In this study we were interested in the HE of the extreme groups of the social hierarchy but other levels could easily be compared following the methods described

To conclude, it is advisable to use methods that take proper account of the survey sampling design so that estimates will be valid not only for the sample used but for the population as a whole. By taking the sampling design into account, the estimates also become unbiased. Also where available, the raw survey data should be used, as it 
contains many more observations and flexible models are readily available for them such as the logistic regression model. The bootstrap method can be used when only aggregated data are available (as in international studies) and the sample sizes (the number of socio-economic levels) are large enough.

\section{Appendix A: Estimation of variance of prevalence of ill health conditions}

The following logistic regression model was fit to the data using the prevalence $(\pi)$ of ill-health conditions as the response variable:

$\operatorname{logit}(\pi)=\beta_{0}+\sum_{j=1}^{N} \beta_{j} * x_{j}$

where $\beta_{\mathrm{j}}, \mathrm{j}=0,1,2, \ldots, \mathrm{N}$ represent parameters to be estimated from the data and $x_{j}, j=1,2,3, N$ represent the various predictor variables that can be incorporated into the model. These variables are the relative position on the socio-economic scale, age, sex and other variables depending on the research question.

The fitted model obtained from (4) is used to predict the prevalence of those at the top and the bottom of the socioeconomic scale for various combinations of the predictor variables; e.g. for males between 25 and 34 years. Using the predicted prevalence and (1), the HE of persons at the top and bottom of the socio-economic scale is obtained.

The variance of the HE is obtained by first determining the variance of the predicted prevalence. The fitted model from (4) provides estimates for the variance $(v)$ of the log odds $(\theta)$ : Thus suppose $\hat{\theta}=\log (\hat{\pi} /(1-\hat{\pi}))$ and $\operatorname{var}(\hat{\theta})=$ $v$, then $\hat{\pi}=\exp (\hat{\theta}) /(1+\exp (\hat{\theta}))$ and the variance of the predicted prevalence according to the Delta method [17] is given by;

$$
\begin{aligned}
\operatorname{Var}(\hat{\pi}) & =\left[\frac{\partial \exp (\hat{\theta}) /(1+\exp (\hat{\theta}))}{\partial \hat{\theta}}\right]^{2} * v \\
& =\left[\exp (\hat{\theta}) /(1+\exp (\hat{\theta}))^{2}\right]^{2} * v
\end{aligned}
$$

\section{References}

1. Bone MR, Bebbington AC, Jagger C, Morgan K, Nicolaas G. Health expectancy and its uses. London: OPCS 1995, 1995.
2. Sanders BS. Measuring community health levels. Am J Public Health 1964;54:1063-70.

3. Sullivan DF. A single index of mortality and morbidity. HSMHA Health Rep. 1971;86:347-54.

4. Robine JM, Jagger C, Romieu I. Disability-free life expectancies for European Union countries. Genus 2001;57:89-101.

5. Wilkins R, Adams OB. Health expectancy in Canada, late 1970s: demographic, regional, and social dimensions. Am J Public Health 1983;73:1073-80.

6. Guralnik JM, Land KC, Blazer D, Fillenbaum GG, Branch LG. Educational status and active life expectancy among older blacks and whites. N Engl J Med. 1993;329:110-6.

7. Sihvonen AP, Kunst AE, Lahelma E, Valkonen T, Mackenbach JP. Socio-economic inequalities in health expectancy in Finland and Norway in the late 1980s. Soc Sci Med. 1998;47:303-15.

8. Cambois E, Robine JM, Hayard M. Social inequality in disability-free life expectancy in the French male population, 19801991. Demography 2001;38:513-24.

9. Bossuyt N, Gadeyne S, Deboosere P, Van Oyen H. Socio-economic inequalities in healthy expectancy in Belgium. Public Health 2004;118:3-10.

10. Perenboom RJM, van Herten LM, Boshuizen HC, van den Bos GAM. Life expectancy without chronic morbidity: trends in gender and socioeconomic disparities. Public Health Rep. 2005; 120:46-54.

11. Hayes L, Berry G. Sampling variability of the Kunst-Mackenbach relative index of inequality. J Epidemiol Community Health 2002;56:762-765.

12. Robine JM, Romieu I, Cambois E. Health expectancy indicators. Bull World Health Organ. 1999;77(2):181-5.

13. Liberatos P, Link BG, Kelsey JL. The measurement of social class in epidemiology. Epidemiol Rev. 1988;10:87.

14. Chiang CL. The life table and its applications. Malabar, FL: Robert E Krieger Publishing Company; 1984.

15. Newman SC. A Markov process interpretation of Sullivan's index of morbidity and mortality. Stat Med. 1988;7:787-94.

16. Pamuk ER. Social-class inequality in infant mortality in England and Wales from 1921 to 1980. Eur J Popul. 1988;4:1-21.

17. Efron B, Tibshirani R. An introduction to the bootstrap. New York: Chapman and Hall; 1993.

18. Oehlert GW. A note on the delta method. Am Stat 1992;46:27-9.

19. Molla M, Wagener D, Madans J. Summary measures of population health: methods for calculating healthy life expectancy. Healthy People 2010, Statistical Notes, 21; 2001

20. van Oyen H, Bossuyt N, Deboosere P, Gadeyene S,Tafforeau J. Differences in health expectancy indicators in Belgium by region. Arch Public Health 2002;60(6):341-62.

21. DeGroot MH () Probability and statistics, 2nd ed. AddisonWesley; 1986. pp. 213-7.

22. van Oyen H, Tafforeau J, Hermans H, Quataert P, Schiettecatte E, Lebrun L Bellamammer. The Belgian health interview survey. Arch Public Health 1997;55:1-13.

23. De Bruin A, Picavet HS, Nosikov A. Health interview surveys: towards international harmonization of methods and instruments. Copenhagen: World Health Organisation; 1996. 